\section{Validation of the Omron HBP-9031C blood pressure monitor for clinics and hospitals according to the ANSI/AAMI/ ISO 81060-2:2013 protocol}

\author{
Kanako Saito ${ }^{1}$, Yukiko Hishiki ${ }^{1}$ and Hakuo Takahashi²* \\ 1Omron Healthcare Co, Ltd, Muko City, Kyoto, 617-0002, Japan \\ 2Department of Cardiology, Biwako Central Hospital, Otsu City, Shiga 520-0834, Japan
}

\section{Abstract}

Objective: The present study aimed to evaluate the accuracy of the Omron HBP-9031C automated oscillometric upper-arm blood pressure (BP) measurement device for blood pressure monitoring, according to the ANSI/AAMI/ISO 81060-2:2013 protocol (ANSI/AAMI/ISO).

Participants and Method: The device evaluations were performed in 85 participants, who fulfilled the inclusion criteria of the protocol. The validation procedure and data analysis followed the protocol precisely.

Results: In the ANSI/AAMI/ISO 81060-2-2013 validation procedure (criterion 1), the mean \pm SD of the differences between the test device and reference BP was $0.5 \pm 7.84 /-1.9 \pm 6.30$ $\mathrm{mmHg}$ (systolic/diastolic). The mean differences between the two observers and the Omron HBP-9031C were $0.5 \pm 6.69 \mathrm{mmHg}$ (range, -18 to $15 \mathrm{mmHg}$ ) for systolic BP and $-1.9 \pm 5.63$ $\mathrm{mmHg}$ (range, -17 to $14 \mathrm{mmHg}$ ) for diastolic BP, according to criterion 2. The two criteria of the ANSI/AAMI/ISO were fulfilled.

Conclusion: The professional OMRON BP monitor, HBP-9031C fulfilled the requirements of the ANSI/AAMI/ISO validation standard and can be recommended for clinical use.

\section{More Information}

*Address for Correspondence: Hakuo Takahashi, MD, PhD, Department of Cardiology, Biwako Central Hospital, Otsu City, Shiga 520-0834, Japan, Tel: +81-77-526-2131; Fax: +81-77-529-0201; Email: takahashi@kou-sei-kai.or.jp

Submitted: 10 August 2019 Approved: 12 August 2019 Published: 13 August 2019

How to cite this article: Saito K, Hishiki Y, Takahashi H. Validation of the Omron HBP9031C blood pressure monitor for clinics and hospitals according to the ANSI/AAMI/ISO 81060-2:2013 protocol. Ann Clin Hypertens. 2019; 3: 043-046.

DOI: 10.29328/journal.ach.1001018

Copyright: (C) 2019 Saito K, et al. This is an open access article distributed under the Creative Commons Attribution License, which permits unrestricted use, distribution, and reproduction in any medium, provided the original work is properly cited

Keywords: Blood pressure monitoring device; Validation study; International Protocol

\section{Introduction}

Monitoring and regulating blood pressure (BP) levels are crucial in the management of cerebral and cardiovascular diseases [1]. However, BP is always fluctuating depending on the effects of environmental factors on autonomic nervous system activity and diurnal variations [2]. Therefore, frequent and repeated measurement of BP under conditions of minimum environmental stress is recommended for the assessment of BP in individuals. For this purpose, fully automated BP monitors are increasingly replacing standard mercury sphygmomanometers [3]. No specific training is required to use these monitors and they avoid observer error. Moreover, mercury sphygmomanometers are no longer distributed on the market due to the possibility of mercury pollution. Furthermore, the white-coat effect is also minimized as healthcare professionals are not present during measurements.

The SPRINT study [4] from 2015 demonstrated novel findings with an automated office BP (AOBP) monitor using the oscillometric method to assess BP levels. In fact, Model 907 (Omron Healthcare) with the oscillometric method was used in that study; BP was measured three times in an isolated room by the patients to avoid the white-coat effect. In the meantime, a systematic review and meta-analysis of 31 articles comprising 9279 participants compared AOBP with awake ambulatory BP, a standard method for predicting cardiovascular risk, was published [5]. Mean AOBP readings were similar to the awake ambulatory BP readings and did not exhibit the "white-coat effect" associated with routine office BP measurements.

Based on the above background, to measure resting BP without white-coat effects by healthcare professionals, even in the clinic or hospital, the Omron HBP-9031C was developed. In the present study, we validated the accuracy of the Omron HBP-9031C according to the international protocols of the American National Standards Institute/ 
Association for the Advancement of Medical Instrumentation/ International Organization for Standardization (ANSI/AAMI/ ISO) 81060-2:2013 standard guidelines [6].

\section{Participants and Methods}

\section{Participants}

All subjects recruited were volunteers. This study was approved by the institutional review board of the Biwako Central Hospital and Omron Healthcare Co., Ltd., with written informed consent obtained from each subject. The study was performed in the measurement room of the Omron Healthcare Co. or Biwako Central Hospital by staff employed by Omron Healthcare Co., Ltd. (Kyoto, Japan). The devices were tested on 89 subjects according to ANSI/AAMI/ISO. In accordance with ANSI/AAMI/ISO, subjects were screened to ensure that sex, age, arm circumference, and BP readings fulfilled the participation requirements described in this protocol. Subjects with arrhythmias, those who moved their arms or bodies during the BP measurements, and those with unclear diastolic BP (DBP) during phase $\mathrm{V}$ of the Korotkoff sounds were excluded from this study.

\section{Methods}

Devices: The Omron HBP-9031C (Omron Healthcare Co, Ltd. Kyoto, Japan) (Figure 1), is designed to measure BP using an oscillometric method on the upper arm by patients in the clinic and hospital, and has a pressure and heart rate range of 0-299 mmHg and 40-180 beats/minute, respectively. Systolic blood pressure (SBP), DBP, and pulse rate are displayed on a liquid crystal digital monitor. The entire weight of the device is approximately $5.5 \mathrm{~kg}$, and the dimensions of the device are approximately $460 \mathrm{~mm} \times 270 \mathrm{~mm} \times 420 \mathrm{~mm}$ (width $\times$ height $\times$ depth). The cuff can be used for arm circumferences ranging from 17-42 $\mathrm{cm}$. Each measurement result of the device can be transferred and stored into a PC via USB, wired or wireless LAN.

Blood pressure measurements: For each experiment, the manufacturer provided standard production device models. The validation team for each device consisted of three observers who were experienced in measuring BP. They also underwent training through the British and Irish

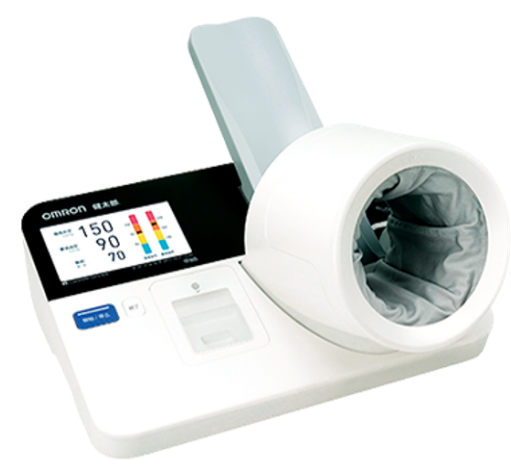

Figure 1: The Omron HBP-9031C BP measuring device.
Hypertension Society's online program (http://www.bihsoc. org). BP measurements were collected by alternating between the Korotkoff method with a mercury sphygmomanometer and the tested device. Simultaneous auscultations were performed by two observers using the double stethoscope (Y tube) when measured with the Korotkoff method. The two observers were blinded to each other's readings, and the third observer served as a supervisor who checked the BP readings.

\section{Procedure}

The subjects were seated in a quiet room at a comfortable room temperature, and they were instructed to avoid talking during the procedure. BP was measured after subjects rested for 10 minutes. The subjects sat on a chair with their legs uncrossed and their feet flat on the floor. The chair had a supportive back as well as elbow and forearm rests. Each subject's arm circumference was measured, and the cuff size was adapted for auscultation. All BP measurements were performed on the subject's left arm at heart level. The devices were validated according to the same-arm, sequential method of the ANSI/AAMI/ISO protocol.

\section{Analysis}

Data analysis was performed according to ANSI/AAMI/ ISO requirements. Data were expressed as a mean \pm standard deviation (SD), and the minimum and maximum values with ranges were calculated. The mean of each pair of observer measurements was calculated as a reference value. The difference between the mean observer value and the test values were calculated according to the protocol and were displayed in Bland-Altman plots against the mean between the two values.

\section{Results}

According to the ANSI/AAMI/ISO guideline, a total of 89 subjects were screened for this experiment. After excluding 4 subjects based on the selection criteria (Table 1), a total of 85 subjects [37 males (44\%) and 48 females (56\%)] fulfilled the inclusion criteria requirements. The mean age was $50 \pm$ 11.4 years (range, 20-74 years). The mean arm circumference was $30.5 \pm 5.7 \mathrm{~cm}$ (range, $19.4-40.3 \mathrm{~cm}$ ). The percentages of subjects were $20.0 \%$ (criteria $\geq 20 \%$ ), $40.0 \%$ ( $\geq 40 \%$ ), $60.0 \%$ ( $\geq 40 \%$ ), and $24.7 \%$ ( $\geq 20 \%$ ) in the arm circumference range of $17.0-23.2 \mathrm{~cm}, 17.0-29.5 \mathrm{~cm}, 29.6-42.0 \mathrm{~cm}$, and $35.9-42.0 \mathrm{~cm}$, respectively (Table 2 ).

\begin{tabular}{|c|c|}
\hline Table 1: Screening and recruitment details. \\
\hline Total screened & 89 \\
\hline Total Excluded & 4 \\
\hline Arrhythmias & 2 \\
\hline Poor quality sounds & 0 \\
\hline Cuff size unavailable & 0 \\
\hline Movement during BP measurement & 0 \\
\hline Blood pressure variation & 2 \\
\hline Total Recruited & 85 \\
\hline
\end{tabular}


Using a standard mercury sphygmomanometer, the mean values of the 255 measurements were $124 \pm 29.5$ (range, $73-$ 193) $\mathrm{mmHg}$ and $76 \pm 17.8$ (range, 39-127) $\mathrm{mmHg}$ for SBP and DBP, respectively.

The percentages for high ( $\geq 160 \mathrm{mmHg}$ ), medium $(\geq 140$ $\mathrm{mmHg}$ ), and low SBP ( $\leq 100 \mathrm{mmHg}$ ) were $11.8 \%$ (criteria $\geq 5 \%$ ), $30.6 \%$ ( $\geq 20 \%$ ), and $24.7 \%$ ( $\geq 5 \%$ ), respectively. The percentages of high $(\geq 100 \mathrm{mmHg}$ ), medium $(\geq 85 \mathrm{mmHg})$, and low DBP ( $\leq 60 \mathrm{mmHg}$ ) were $7.8 \%$ (criteria $\geq 5 \%$ ), $35.7 \%$ ( $\geq 20 \%$ ) and $20.8 \%(\geq 5 \%)$, respectively (Table 3 ).

The differences between the measurements recorded by two observers were $0 \pm 1.4 \mathrm{mmHg}$ and $0 \pm 1.7 \mathrm{mmHg}$ for SBP and DBP, respectively. The mean differences between the values recorded by two observers and the Omron HBP-9031C were $0.5 \pm 7.84 \mathrm{mmHg}$ (range, -23 to $19 \mathrm{mmHg}$ ) and $-1.9 \pm 6.30$ $\mathrm{mmHg}$ (range, -19 - $16 \mathrm{mmHg}$ ) for SBP and DBP, respectively, according to criterion 1 . These data fulfilled the ANSI/AAMI/ ISO requirements of $\leq 5 \pm \leq 8 \mathrm{mmHg}$. The mean differences between the values recorded by two observers and the Omron HBP-9031C were $0.5 \pm 6.69 \mathrm{mmHg}$ (range, $-18-15 \mathrm{mmHg}$ ) and $-1.9 \pm 5.63 \mathrm{mmHg}$ (range, $-17-14 \mathrm{mmHg}$ ) for SBP and DBP, respectively, according to criterion 2 . Thereby, the SD is calculated to be less than $6.92 \mathrm{mmHg}$ and $6.68 \mathrm{mmHg}$ for SBP and DBP, respectively, according to criterion l. These results are in accordance with the ANSI/AAMI/ISO requirements for criteria 1 and 2. Therefore, the Omron HBP-9031C fulfills the validation criteria of the ANSI/AAMI/ISO requirements (Table 4).

\begin{tabular}{|c|c|}
\hline \multicolumn{2}{|l|}{ Table 2: Baseline characteristics of the subjects. } \\
\hline Women (\%) & 56 \\
\hline Age (years) & $50 \pm 11.4(20-74)$ \\
\hline Arm circumference (cm) & $30.5 \pm 5.7(19.4-40.3)$ \\
\hline \multicolumn{2}{|c|}{ Limb size distribution } \\
\hline $17.0-23.2 \mathrm{~cm}(\%)$ & 20.0 \\
\hline $17.0-29.5 \mathrm{~cm}(\%)$ & 40.0 \\
\hline $29.6-42.0 \mathrm{~cm}(\%)$ & 60.0 \\
\hline $35.9-42.0 \mathrm{~cm}(\%)$ & 24.7 \\
\hline
\end{tabular}

\begin{tabular}{|c|c|}
\hline Table 3: Blood pressure distribution. \\
\hline SBP $(\mathrm{mmHg})$ & $124 \pm 29.5(73-193)$ \\
\hline $160 \mathrm{mmHg}(\%)$ & 11.8 \\
\hline$\geq 140 \mathrm{mmHg}(\%)$ & 30.6 \\
\hline$\leq 100 \mathrm{mmHg}(\%)$ & 24.7 \\
\hline $\mathrm{DBP}(\mathrm{mmHg})$ & $76 \pm 17.8(39-127)$ \\
\hline$\geq 100 \mathrm{mmHg}(\%)$ & 7.8 \\
\hline$\geq 85 \mathrm{mmHg}(\%)$ & 35.7 \\
\hline$\leq 60 \mathrm{mmHg}(\%)$ & 20.8 \\
\hline
\end{tabular}

\begin{tabular}{|c|c|}
\hline \multicolumn{2}{|c|}{ Table 4: Validation results. } \\
\hline & Criterion 1 \\
\hline & $0.5 \pm 7.84(-23-19)$ \\
\hline SBP $(\mathrm{mmHg})$ & $-1.9 \pm 6.30(-19-16)$ \\
\hline DBP $(\mathrm{mmHg})$ & Criterion 2 \\
\hline & $0.5 \pm 6.69(-18-15)$ \\
\hline SBP $(\mathrm{mmHg})$ & $-1.9 \pm 5.63(-17-14)$ \\
\hline DBP $(\mathrm{mmHg})$ & \\
\hline
\end{tabular}

Figure 2A and B show the differences in the SBP and DBP readings relative to the mean differences between the HBP9031C and mercury sphygmomanometer measurements.

\section{Discussion}

In the present study, we demonstrated that the Omron HBP-9031C, which is a desktop-type oscillometric BP monitor, fulfilled the validation criteria of ANSI/AAMI/ IS081060-2:2013 guidelines, and is proposed as a useful tool in the clinic and hospital.

Several organizations, such as the US Association for the Advancement of Medical Instrumentation (AAMI), the British Hypertension Society, the European Society of Hypertension (ESH), Working Group on Blood Pressure (BP) Monitoring, and the International Organization for Standardization (ISO), have been developing a single protocol for universal clinical validation of BP measuring devices [7]. In the discussion, a majority of the ANSI/AAMI/ISO81060-2:2013 protocol is highly regarded. Therefore, the present results of this validation study, by the ANSI/AAMI/IS081060-2:2013 guidelines, are also highly appraised.

The Omron HBP-9031C is an installation-type BP monitor, which measures BP by the insertion of one's arm through the cuff hoop and pressing the start button. The positioning of the arm is guided by the illumination of a green signal light when placed properly. The oscillometric method used in this device senses the pulses oscillations due to cuff pressure regardless of the position of the cuff around the upper arm, making it resistant to environmental factors except for arm movements. Since measurements with this device are very easy to manipulate, healthcare professionals must not be present when BP is measured to avoid white-coat effects. The results can be printed out and given to the attending doctor in the clinic or hospital. Those BP measurements will be superior to the traditional office BP measurements for the clinical management and control of BP.

\section{Conclusion}

The results of this study show that Omron HBP-9031C devices met the requirements of the ANSI/AAMI/ISO protocol, and may be useful for BP measurement in the clinic and hospital.
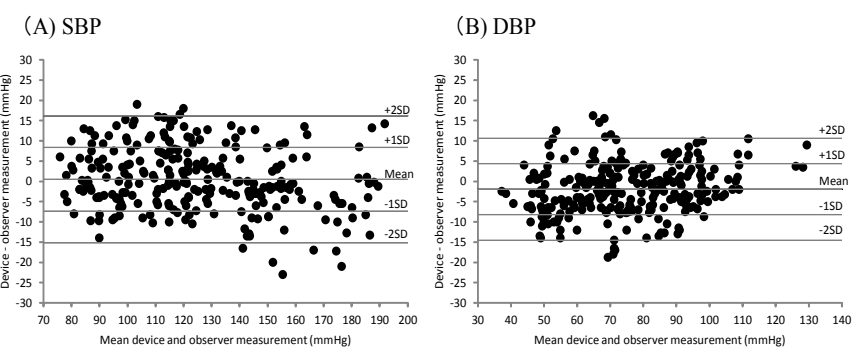

Figure 2: Bland-Altman plots of the differences between Omron HBP-9031C readings and observer measurements. (A) Systolic blood pressure (SBP), and (B) diastolic blood pressure (DBP) according to ANSI/AAMI/ISO protocols. 


\section{Acknowledgement}

We would like to thank Editage (www.editage.com) for English language editing.

\section{References}

1. Ikeda $\mathrm{N}$, Inoue $M$, Iso $H$, Ikeda $S$, Satoh $T$, et al. Adult mortality attributable to preventable risk factors for non-communicable diseases and injuries in Japan: a comparative risk assessment. PLoS Med. 2012; 9: e1001160. PubMed: https://www.ncbi.nlm.nih.gov/ pubmed/22291576

2. Zhang $Y$, Agnoletti $D$, Blacher J, Safar ME. Blood pressure variability in relation to autonomic nervous system dysregulation: the X-CELLENT study. Hypertens Res. 2012; 35: 399-403. PubMed: https://www.ncbi. nlm.nih.gov/pubmed/22129516

3. O'Brien E, Dolan E, Stergiou GS. Achieving reliable blood pressure measurements in clinical practice: It's time to meet the challenge. $J$ Clin Hypertens (Greenwich). 2018; 20: 1084-1088. PubMed: https:// www.ncbi.nlm.nih.gov/pubmed/30003700
4. SPRINT Research Group, Wright JT Jr, Williamson JD, Whelton PK, Snyder JK, et al. A Randomized Trial of Intensive versus Standard Blood-Pressure Control. N Engl J Med. 2015; 373: 2103-2116.

5. Roerecke M, Kaczorowski J, Myers MG. Comparing Automated Office Blood Pressure Readings with Other Methods of Blood Pressure Measurement for Identifying Patients With Possible Hypertension: A Systematic Review and Meta-analysis. JAMA Intern Med. 2019; 179: 351-362. PubMed: https://www.ncbi.nlm.nih.gov/pubmed/30715088

6. Association for the Advancement of Medical Instrumentation American National Standard. ANSI/AAMI/ISO 81060-2:2013 Non-invasive Sphygmomanometers - Part 2: Clinical investigation of automated measurement type. 2019.

7. Stergiou GS, Alpert B, Mieke S, Asmar R, Atkins N, et al. A universal standard for the validation of blood pressure measuring devices: Association for the Advancement of Medical Instrumentation/European Society of Hypertension/International Organization for Standardization (AAMI/ESH/ISO) Collaboration Statement. J Hypertens. 2018; 36: 472478. PubMed: https://www.ncbi.nlm.nih.gov/pubmed/29384983 